\title{
Drug Resistance and Epigenetic Modulatory Potential of Epigallocatechin-3-Gallate Against Staphylococcus Aureus
}

\section{Ana Sofia Zeferino}

Centro Hospitalar de Lisboa Central EPE

\section{Ana Rita Mira}

Hospital do Espirito Santo Evora

\section{Mariana Delgadinho}

Heatlh and Technology Recearch Center ESTeSL IPL

\section{Miguel Brito}

Instituto Politecnico de Lisboa Escola Superior de Tecnologia da Saude de Lisboa

\section{Tomás Ponte}

Hospital Garcia de Orta EPE

Edna Ribeiro ( $\nabla$ edna.ribeiro@estesl.ipl.pt)

Instituto Politecnico de Lisboa Escola Superior de Tecnologia da Saude de Lisboa

\section{Research}

Keywords: S. aureus colonization, Epigallocatechin-3-gallate (EGCG), antibiotic resistance, synergistic effects, epigenetic modulators, drug resistance genes

Posted Date: June 11th, 2021

DOI: https://doi.org/10.21203/rs.3.rs-606371/v1

License: (c) (i) This work is licensed under a Creative Commons Attribution 4.0 International License. Read Full License

Version of Record: A version of this preprint was published at Current Microbiology on April 9th, 2022. See the published version at https://doi.org/10.1007/s00284-022-02841-5. 


\section{Abstract}

Background: Antimicrobial resistance of human pathogens such as methicillin-resistant Staphylococcus aureus (MRSA), is described by the World Health Organization as a health global challenge and efforts must be made for the discovery of new effective and safe compounds. This work aims to evaluate Epigallocatechin-3-gallate (EGCG) epigenetic and drug modulatory potential against $S$. aureus in vitro and in vivo.

Methods: S. aureus strains were isolated from commensal flora of healthy volunteers. Antibiotic susceptibility and synergistic assay were assessed trough disk diffusion accordingly to EUCAST guidelines with and without coexposure to EGCG at final concentrations of 250,100, 50 and $25 \mu \mathrm{g} / \mathrm{ml}$. Transcriptional expression of orf $x$, spdC and WalKR was performed trough qRT-PCR. A 90 days interventional study was performed with a daily consumption of $225 \mathrm{mg}$ of EGCG.

Results: Obtained data revealed a high prevalence of $S$. aureus colonization in health care workers and clearly demonstrate the antimicrobial and synergistic potential of EGCG as well as divergent resistant phenotypes associated with altered transcriptional expression of epigenetic and drug response modulators genes.

Conclusions: Here we demonstrate the potential of EGCG for antimicrobial treatment and/or therapeutic adjuvant against antibiotic resistant microorganisms and report divergent patterns of epigenetic modulators expression associated with phenotypic resistance profiles.

\section{Introduction}

The World Health Organization (WHO) describes antimicrobial resistance in human pathogens as a global health challenge (1). For the past decades, with the global escalation in the development of antibiotic resistant microorganisms (2) both social and scientific concerns have emerged regarding the intensive prescription of antibiotics and the development of pathogens with resistant phenotypes (3).

Airborne microorganisms such as Staphylococcus aureus, are one of these pathogens. Some $S$. aureus strains are resistant to multiple antibiotics, which difficult the treatment of infections caused by this pathogen, and great attention has been given to methicillin-resistant $S$. aureus (MRSA). During its evolution, $S$. aureus acquired, via horizontal genetic transfer, the Staphylococcal Cassette Chromosome mec complex (SCCmec), in which is inserted the $m e c A$ gene encoding the modified penicillin-binding protein 2 (PBP2), responsible for the resistance to $\beta$-lactam antibiotics (oxacillin, streptomycin, tetracycline, erythromycin, among others.), by decreasing the affinity of the binding of antibiotics to their receptor in the bacterium (4-6). MRSA is one of the most important human pathogens, being a major cause of infections worldwide (7) associated with extremely high mortality rates for invasive blood-stream and pneumonic infections (8). Additionally, the ability to become part of the human commensal flora is also a potentially relevant hazard since human MRSA carriers have increased risk for subsequent occurrence of clinical disease (up to 10-fold) and become a bacterial reservoir with associated high risk to transfer the infection to others or contaminate foods and food surfaces during handling (9). Currently it is estimated that $S$. aureus colonizes transiently or persistently approximately $30 \%$ of the human population asymptomatically, in which the highly variable nasal microbiota plays a key role (10). Moreover, high nasal colonization by MRSA among health professionals is considered a major issue in order to prevent nosocomial infections (11). Although most of the studies performed regarding $S$. aureus epidemiology have been focused on the emergence and dissemination of MRSA strains in healthcare settings, currently much attention is also given for dissemination in the community (12). 
Considering the relevance of this pathogen for clinical setting and the community and general as well as the development of new resistant strains, the identification of new compounds with anti-microbiological potential and resistance reversion is crucial. Relevantly, reversal of the resistance phenotype of this microorganism has been suggested in some in vitro studies associated with exposure to Epigallocatechin-3-gallate (EGCG), the largest constituent of green tea (13). Synergism between EGCG and $\beta$-lactams has been focused on $S$. aureus, in particular MRSA and showed that EGCG can damage the bacterial cell wall, compromising its integrity as it binds to peptidoglycan $(14,15)$. Roccaro et al. proved that catechins interact synergistically with tetracycline against $S$. aureus (15) and different studies have also demonstrated a synergistic effect with penicillin, oxacillin, ampicillin / sulbactam and imipenem in MRSA (13,15-17).

Epigallocatechin-3-gallate (EGCG) is able to affect human plasma profile (18) (and associated health benefits have been related to their epigenetic effects as these compounds are able to target both histone acetyl transferases (HATs) and histone deacetylases (HDACs), regulate acetylation of histones and non-histone chromatin proteins and affect DNA methylation $(19,20)$ In studies related to carcinogenesis, EGCG ability to induce epigenetic reactivation of silenced genes or epigenetic inhibition of oncogenes has been associated with the inhibition of DNMTs or the activity of HDACs (20). In Staphylococci the orfx gene encodes a conserved staphylococcal ribosomal methyltransferase, produced constitutively during bacterial growth, inserted in the SCCmec complex which contains the mecA gene, responsible for resistance to $\beta$-lactams in MRSA (21). Moreover, the WalKR system is a two-component system, which upregulates $s p d C$ gene expression, considered a new virulence factor in $S$. aureus (22), known to be crucial for the rapid adaptation of $S$. aureus to a wide range of environmental conditions and modulate drug resistance (22-25).

This work aims to assess $S$. aureus methicillin-resistant (MRSA) and sensible (MSSA) strains colonization prevalence in health care workers, potential epigenetic and drug response transcriptional modulator effects and to evaluate the potential of EGCG in reversing MRSA phenotype in vivo. This study is intended to demonstrate in vitro and in vivo EGCG potential for antimicrobial treatment and/or therapeutic adjuvant against resistant microorganisms.

\section{Materials And Methods}

\subsection{Study population and design}

For in vitro assay, $S$. aureus strains were isolated from occupationally exposed healthcare workers $(n=38)$. For the in vivo human assay, we performed an interventional, uncontrolled, prospective, longitudinal and of individual analysis study, which included 30 healthy individuals (with no previously diagnosed pathologies). Inclusion criteria considered were adult voluntaries (ages superior to 18 years old and less than 65 years old) with no acknowledged previously diagnosed pathology of any type. Exclusion criteria applied included viral infections, consumption of tea and forgotten capsules on consecutive days during the study. Data was analyzed under blind conditions.

\subsection{Supplementation and questionnaires}

Human clinical trials demonstrated that $400 \mathrm{mg}$ and $800 \mathrm{mg}$ of EGCG intake (26), results in peak serum concentrations in the range of 100 to $400 \mathrm{ng} / \mathrm{ml}$ (27) with no reported severe secondary effects. Commercial capsules of green tea extract with $\left.225 \mathrm{mg} \mathrm{EGCG/capsule} \mathrm{(My} \mathrm{Protein}{ }^{\circledR}\right)$ were provided for all the participants in the study with the instructions to take 1 capsule daily. EGCG dosage was selected considering that, $400 \mathrm{mg}$ and $800 \mathrm{mg}$ of EGCG intake, which are considered safe doses previously used in human clinical trials. Thus, the selected dosage is considered safe. The participants filled up 2 questionnaires, the first in the moment of the first specimen collection (T0, June) with questions regarding demographic data such as age, gender, weight (cm), height (kg) and smoking habits. In the second questionnaire, after 90 days (T90, September) of the interventional study, the participants were 
asked to report adverse effects through the intervention, namely alterations in the nervous system (headaches, migraines, mood swings), gastrointestinal system (heartburn, reflux, diarrhea, cramps, weight loss / gain), cardiac and respiratory effects and information regarding potential missing capsules intake.

\subsection{Biological samples:}

For isolation of $S$. aureus from commensal flora, biological samples were obtained through nasopharyngeal swab procedure using transport swabs with Stuart media, and immediately transported to the laboratory.

For in vivo assessment of EGCG exposure effect, biological samples were collected at time 0 (T0) followed by 90 days, of ingestion of commercial capsules of green tea extract (225mg EGCG / capsule) daily. After 90 days of exposure a second biological sampling through nasopharyngeal swab was performed (T90).

\subsection{S. aureus identification}

For $S$. aureus identification, biological samples were inoculated in Columbia agar with $5 \%$ sheep blood non-selective media as well as in selective media CHROMID ${ }^{\circledR}$ MRSA, and incubated for 24 and 48 hours at $37^{\circ} \mathrm{C}$. After incubation, $S$. aureus suspicious colonies were isolated and incubated for 24 hours at $37^{\circ} \mathrm{C}$. Identification of $S$. aureus was performed trough catalase test and Slidex Staph Kit (Biomerieux ref \#73115). MRSA strains were identified through Slidex MRSA detection Test Kit (Biomerieux ref \#73117). In this work positive (S. aureus MRSA laboratory collection) and negative (S. aureus ATCC 25923) control strains where be included as positive and negative controls.

\subsection{Antimicrobial assay}

S. aureus strains isolated from commensal flora were inoculated in $1 \mathrm{ml}$ of Mueller Hinton at 0.5 McFarland turbidity. Antibiotic susceptibility was assessed trough disk diffusion method seeded in Mueller Hinton Agar with commercial discs of amoxicillin $(25 \mu \mathrm{g})$, tetracycline $(30 \mu \mathrm{g})$, gentamicin $(30 \mu \mathrm{g})$ and imipenem $(10 \mu \mathrm{g})$. After incubation of $18 \mathrm{~h}, 24$ $\mathrm{h}$ and $48 \mathrm{~h}$ at $37^{\circ} \mathrm{C}$, antibiotic disk zone of inhibition was measured, and susceptibility assessed using EUCAST Clinical Breakpoint Tables v. 10.0, valid from 2020-01-01 (28) data summarized in table 1.

Table 1 - Inhibition zone intervals to determine sensitivity or resistance to antibiotics, according to values in the EUCAST v10.0 table valid from 01-01-2020 (28)

\begin{tabular}{|llc|}
\hline & \multicolumn{2}{l|}{ Zone of inhibition (mm) } \\
\hline Antibiotics & Sensible & Resistant \\
\hline Imipenem $10 \mu \mathrm{g}^{\star}$ & $\geq 22$ & $<22$ \\
\hline Tetracycline $30 \mu \mathrm{g}$ & $\geq 22$ & $<19$ \\
Gentamicin $30 \mu \mathrm{g}$ & $\geq 18$ & $<18$ \\
\hline amoxicillin $25 \mu \mathrm{g}^{\star}$ & $\geq 22$ & $<22$ \\
\hline
\end{tabular}

* The values of the measurement intervals of the zones of inhibition for imipenem and amoxicillin were inferred from the values of cefoxitin, according to the indications in the EUCAST v10.0 table valid from 01-01-2020.

\subsection{Synergy evaluation}

To assess the synergistic potential of EGCG (E4143 Sigma), S. aureus strains isolated from commensal flora and infections were inoculated in $1 \mathrm{ml}$ of Mueller Hinton Broth with EGCG at final concentrations of $250 \mu \mathrm{g} / \mathrm{ml}, 100 \mu \mathrm{g} / \mathrm{ml}$, 
$50 \mu \mathrm{g} / \mathrm{ml}$ and $25 \mu \mathrm{g} / \mathrm{ml}$ at $0.5 \mathrm{McFarland}$ turbidity. Antibiotic susceptibility was assessed trough disk diffusion method seeded in Mueller Hinton Agar with commercial discs of amoxicillin $(25 \mu \mathrm{g})$, tetracycline $(30 \mu \mathrm{g})$, gentamicin $(30 \mu \mathrm{g})$ and imipenem $(10 \mu \mathrm{g})$. (positive control). After incubation of $18 \mathrm{~h}, 24 \mathrm{~h}$ and $48 \mathrm{~h}$ at $37^{\circ} \mathrm{C}$, antibiotic disk zone of inhibition was measured and susceptibility assessed using EUCAST Clinical Breakpoint Tables v. 10.0, valid from 2020-01-01 (28). The significant differences between the different times of exposure, MSSA and MRSA strains were assessed using Student's t-test. P-Values < 0.02 were considered significant for EGCG exposure per se and P-Values < 0.01 were considered significant for EGCG co-exposure with Imipenem, Tetracycline, Gentamycin and Amoxicillin.

\subsection{Transcriptional expression analysis}

For the transcriptional analysis study, MSSA and MRSA strains with divergent resistance profiles were selected.

Cell lysates were used for extraction of bacteria total RNA using the NZY Total RNA Isolation kit (Nzytech), according to the manufacturer's protocol. RNA samples concentration was determined using the Qubit ${ }^{\text {TM }}$ RNA HS Assay Kit in Qubit $^{\text {TM }}$ 3.0 Fluorometer (Invitrogen) and $1 \mu \mathrm{g}$ of total RNA was reverse transcribed to cDNA by means of random hexamers from the RevertAid RT Kit (ThermoScientific). Quantitative real-time PCR (qRT-PCR) was performed on a CFX Connect ${ }^{\mathrm{TM}}$ Real-Time PCR Detection System (Bio-rad) using specific primers, listed in Table 2, for the genes orf $x$, spdC and WalKR and 16S rRNA was used as reference gene for data normalization. Reactions were performed in triplicate using the iQ SYBR Green Supermix (Bio-rad) in a final volume of $20 \mu l$. Additionally, control PCRs were performed for all primer combinations without template. The utilized cycling conditions were: initial activation of $95^{\circ} \mathrm{C}$ for $2 \mathrm{~min}$ followed by 40 cycles of $95^{\circ} \mathrm{C}$ for $30 \mathrm{~s}, 55^{\circ} \mathrm{C}$ for $30 \mathrm{~s}$, and $72{ }^{\circ} \mathrm{C}$ for $40 \mathrm{~s}$. Potential primer-dimer formation and contaminations were also assessed and excluded. Relative quantification was undertaken by normalizing threshold cycles (Ct) of the target genes with the mean $\mathrm{Ct}$ of $16 \mathrm{~S}$ rRNA. Transcription levels were assessed by calculating $\Delta \Delta \mathrm{Ct}(\Delta \Delta \mathrm{Ct}=\Delta \mathrm{Ct}$ resistant phenotypes-mean $\Delta \mathrm{Ct}$ most resistant strains (control)).

Table 2 - Primer sequences, accession numbers and product lengths for qRT-PCR analysis.

\begin{tabular}{|llll|}
\hline Genes & Forward primer $\left(\mathbf{5}^{\prime} \rightarrow \mathbf{3}^{\prime}\right)$ & Reverse Primer $\left(\mathbf{3}^{\prime} \rightarrow \mathbf{5}^{\prime}\right)$ & Reference \\
\hline Orfx & GGGCAAAGCGACTTTGTATT & TGGGAATGTCATTTTGCTGA & AAW81344.1* \\
\hline SpdC & GCAGTAGGATACATTGGTT & CAGCCTCAGTATGATTAGTT & 22 \\
\hline WalKR & GTGTACTGTGCATACGATGGTAATGATGC & CGTTACATAGTCATCTGCACCTAGTTCTA & 22 \\
\hline $\begin{array}{l}16 S \\
\text { rRNA }\end{array}$ & ACGTGGATAACCTACCTATAAGACTGGGAT & TACCTTACCAACTAGCTAATGCAGCG & 22 \\
\hline
\end{tabular}

*GenBank accession numbers (National Center for Biotechnology).

\subsection{Statistical analysis}

All statistical calculations were performed by using IBM SPSS statistics 22 software. The significant differences between different groups were analysed by a Student's t-test (comparison for two groups) and $p<0.01$ was considered statistically significant. Results are presented as mean \pm standard deviation.

\subsection{Ethics statement}

This work is included in two projects from the Instituto Politécnico de Lisboa accepted in Escola Superior de Tecnologia da Saúde ethical council ref: CE-ESTeSL-Nº.18-2019 and CE-ESTeSL-Nº. 20-2020. All volunteers provided a 
signed written informed consent before enrolment in the study in accordance with the Helsinki Declaration and Oviedo Convention and in Agreement with the Portuguese law n 58/2019 de 8 de agosto regarding data protection.

\section{Results}

3.1. Healthcare workers presented high prevalence levels of S. aureus colonization with assoiated divergent resistant phenotypes and EGCG efefcts

The prevalence of $S$. aureus in the population studied in samples collected from healthcare workers $(n=38)$ was $42.1 \%$ (16/38), of which $18.4 \%(7 / 38)$ were MSSA and $23.7 \%(9 / 38)$ MRSA.

3.1.1. S. aureus (MSSA and MRSA) commensal strains presented divergent resistant phenotypes against imipenem, tetracycline, gentamycin and amoxicillin.

Phenotyping of the antibiotic resistance profile of strains isolated from samples collected from volunteers with healthcare occupational exposure, was performed regarding sensitivity to imipenem $10 \mu \mathrm{g}$, tetracycline $30 \mu \mathrm{g}$, gentamicin $30 \mu \mathrm{g}$ and amoxicillin $25 \mu \mathrm{g}$ in order to identify divergent profiles. After incubation $\left(24 \mathrm{~h}\right.$ at $\left.37^{\circ} \mathrm{C}\right)$, the antibiotic inhibition zones were measured for each strain, and the sensible (S) and resistant (R) characterization was performed according to the values established by EUCAST and the results obtained are recorded in Table 3. Data showed that all strains are sensible to tetracycline and gentamicin. MSSA strains encoded as CC4, MB1, MB6, MB10 and MRSA strains encoded with MB2, MB4, VFXB14, VFXB15, VFXB16 were resistant to amoxicillin. The coded strain MB12 was resistant to both imipenem and amoxicillin.

Table 3 - Resistance profile divergence of isolated MSSA and MRSA strains to Imipenem (10 $\mathrm{gg})$, Tetracycline (30 $\mu \mathrm{g})$, Gentamycin $(30 \mu \mathrm{g})$ and Amoxicillin $(25 \mu \mathrm{g})$. 


\begin{tabular}{|c|c|c|c|c|c|}
\hline & Code & IMIP 10 & TETRA 30 & GENTA 30 & AMOX 25 \\
\hline & CC3 & $S$ & $S$ & $S$ & $S$ \\
\hline M & CC4 & S & S & S & $\mathbf{R}$ \\
\hline s & MB1 & $s$ & $s$ & $s$ & $\mathbf{R}$ \\
\hline s & MB6 & $S$ & $S$ & $S$ & $\mathbf{R}$ \\
\hline \multirow[t]{5}{*}{ A } & MB10 & S & S & S & $\mathbf{R}$ \\
\hline & MB17 & s & s & $\mathrm{s}$ & s \\
\hline & $5-7 S$ & S & S & S & S \\
\hline & MB2 & S & S & S & $\mathbf{R}$ \\
\hline & MB4 & s & $\mathrm{s}$ & $\mathrm{s}$ & $\mathbf{R}$ \\
\hline M & MB5 & S & $\mathrm{S}$ & $\mathrm{S}$ & S \\
\hline $\mathrm{R}$ & MB12 & $\mathbf{R}$ & S & S & $\mathbf{R}$ \\
\hline s & $1-2 S$ & $S$ & $S$ & $S$ & $S$ \\
\hline \multirow[t]{4}{*}{ A } & $V F X B 7$ & $S$ & $S$ & $S$ & $S$ \\
\hline & VFXB14 & S & $S$ & $S$ & $\mathbf{R}$ \\
\hline & VFXB15 & s & S & S & $\mathbf{R}$ \\
\hline & VFXB16 & S & $S$ & S & $\mathbf{R}$ \\
\hline
\end{tabular}

3.1.2. Exposure to EGCG induce differential synergistic effects in MSSA and MRSA resistant phenotypes

In order to assess potential synergism divergence between EGCG and the different antibiotics tested, isolated strains were inoculated at different concentrations of EGCG $(250 \mu \mathrm{g} / \mathrm{ml}, 100 \mu \mathrm{g} / \mathrm{ml}, 50 \mu \mathrm{g} / \mathrm{ml}$ and $25 \mu \mathrm{g} / \mathrm{ml})$ and antibiotic resistance tests performed.

Regarding the results obtained with imipenem, the MRSA strain encoded as MB12, which showed a resistant phenotype (Table 3), when exposed to different concentrations of EGCG, the phenotype went from resistant to sensible. EGCG potentiated the action of imipenem and the phenotype reversal was observed after co-exposure with EGCG at final concentrations of $250 \mu \mathrm{g} / \mathrm{ml}, 100 \mu \mathrm{g} / \mathrm{ml}, 50 \mu \mathrm{g} / \mathrm{ml}$ and $25 \mu \mathrm{g} / \mathrm{ml}$.

On the other hand, amoxicillin MSSA and MRSA resistant strains namely CC4, MB1, MB6, MB10, MB2, MB4, MB12, VFXB14 VFXB15, VFXB16 after exposure with EGCG presented divergent resistance phenotypes which are summarized in Table 4. Strains encoded as MB6, MB2, MB4, when exposed to EGCG in different concentrations, the resistance phenotype reverted to sensible, indicating that EGCG potentiated the action of amoxicillin in these strains. Strains encoded as MB1 and VFXB16 only demonstrated the reversion of the resistance to sensible phenotype at

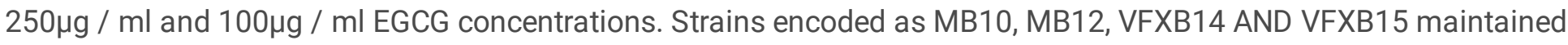
the resistance phenotype, that is, in these strains there was no synergism between EGCG and amoxicillin.

Table 4- Resistance profile of isolated MSSA and MRSA strains to resistant to Amoxicillin ( $25 \mu \mathrm{g})$ after co-exposure with EGCG at final concentrations of $250 \mu \mathrm{g} / \mathrm{ml}, 100 \mu \mathrm{g} / \mathrm{ml}, 50 \mu \mathrm{g} / \mathrm{ml}$ and $25 \mu \mathrm{g} / \mathrm{ml}$. 


\begin{tabular}{|c|c|c|c|c|c|c|}
\hline & Code & AMOX & $\begin{array}{l}\mathrm{AMOX}+250 \mu \mathrm{g} / \mathrm{ml} \\
\mathrm{EGCG}\end{array}$ & $\begin{array}{l}\text { AMOX + } 100 \mu \mathrm{g} / \mathrm{ml} \\
\text { EGCG }\end{array}$ & $\begin{array}{l}\text { AMOX }+50 \mu \mathrm{g} / \mathrm{ml} \\
\text { EGCG }\end{array}$ & $\begin{array}{l}\text { AMOX + } 25 \mu \mathrm{g} / \mathrm{ml} \\
\text { EGCG }\end{array}$ \\
\hline M & CC4 & $\mathbf{R}$ & $S$ & $S$ & $S$ & $\mathbf{R}$ \\
\hline$S$ & MB1 & $\mathbf{R}$ & $S$ & $s$ & $\mathbf{R}$ & $\mathbf{R}$ \\
\hline S & MB6 & $\mathbf{R}$ & $S$ & $S$ & $S$ & $S$ \\
\hline \multirow[t]{2}{*}{ A } & MB10 & $\mathbf{R}$ & $\mathbf{R}$ & $\mathbf{R}$ & $\mathbf{R}$ & $\mathbf{R}$ \\
\hline & MB2 & $\mathbf{R}$ & $S$ & $S$ & $S$ & $S$ \\
\hline M & MB4 & $\mathbf{R}$ & $S$ & $S$ & $S$ & $S$ \\
\hline $\mathrm{R}$ & MB12 & $\mathbf{R}$ & $\mathrm{R}$ & $\mathbf{R}$ & $\mathbf{R}$ & $\mathbf{R}$ \\
\hline S & VFXB14 & $\mathbf{R}$ & $\mathbf{R}$ & $\mathbf{R}$ & $\mathbf{R}$ & $\mathbf{R}$ \\
\hline \multirow[t]{2}{*}{ A } & VFXB15 & $\mathbf{R}$ & $\mathbf{R}$ & $\mathbf{R}$ & $\mathbf{R}$ & $\mathbf{R}$ \\
\hline & $V F X B 16$ & $\mathbf{R}$ & $S$ & $S$ & $\mathbf{R}$ & $\mathbf{R}$ \\
\hline
\end{tabular}

3.1.3. Imipenem, tetracycline, gentamycin and amoxicillin inibition zones values are affected by EGCG exposure

Regarding time exposure effects in EGCG co-exposure with the tested antibiotics, we observed significant divergence in MSSA with Imipenem and Amoxicillin between $18 \mathrm{~h}$ and $24 \mathrm{~h}$ exposure $(\mathrm{p}=0,00190135$ and $p=1,5058 \mathrm{E}-05$, respectively) and $24 h$ and $48 h(p=0,014651$ and $p=0,000525$, respectively).

MRSA between $18 \mathrm{~h}$ and $24 \mathrm{~h}$ exposure in Imipenem and Amoxicillin ( $p=3,27033 \mathrm{E}-05$ and 0,049234613 respectively) and $24 \mathrm{~h}$ to $48 \mathrm{~h}$ only in Amoxicillin $(\mathrm{p}=0,000159)$. On the other hand, at the same exposure time we also reported differences between MSSA and MRSA strains namely after 18h EGCG co-exposure with Imipenem and Amoxicillin ( $p=$ $2,87778 \mathrm{E}-07$ and $p=0,000201292$, respectively) and Imipenem for $24 \mathrm{~h}$ and $48 \mathrm{~h}$ exposures ( $p=8,04449 \mathrm{E}-07$ and 6,5191E-06, respectively).

3.1.4. Epigenetic and drug resistance modulators expression patterns differ in divergent resistance phenotypes strains after EGCG exposure

Expression analysis of staphylococci methyltransferase (orf $x$ ) and drug resistance (spdC and WalKR) genes was performed in selected MSSA and MRSA strains with described divergent resistance phenotypes obtained after EGCG exposure. Results are summarized in table 5. MSSA strain MB10 and MRSA strain VFXB14 were the most resistant strains and so utilized for transcriptional expression comparation in relation to the other selected strains.

Table 5 - MSSA and MRSA selected strains code and resistant phenotype regarding (imipenem (IMIP), tetracycline (TETRA), gentamycin (GENTA) and amoxicillin (AMOX)) selected for transcriptional analysis. 


\begin{tabular}{|ll|}
\hline Code & Resistance phenotype \\
\hline CC4 & IMIP S, TETRA S, GENTA S, AMOX R reversion up to $50 \mu \mathrm{g} / \mathrm{ml}$ EGCG \\
\hline MB6 & IMIP S, TETRA S, GENTA S, AMOX R total reversion with ECGC \\
\hline MB10 & IMIP S, TETRA S, GENTA S, AMOX R no reversion \\
\hline MB17 & IMIP S, TETRA S, GENTA S, AMOX S \\
\hline MB2 & IMIP S, TETRA S, GENTA S, AMOX R total reversion with ECGC \\
\hline MB12 & IMIP R total reversion, TETRA S, GENTA S, AMOX R no reversion \\
\hline MB5 & IMIP S, TETRA S, GENTA S, AMOX S \\
\hline VFXB14 & IMIP S, TETRA S, GENTA S, AMOX R no reversion \\
\hline VFXB16 & IMIP S, TETRA S, GENTA S, AMOX R reversion up to $100 \mu \mathrm{g} / \mathrm{ml} \mathrm{EGCG}$ \\
\hline
\end{tabular}

3.1.5. Orfx, spdC and WalKR expression levels are affected by co-exposure with EGCG in selected MSSA and MRSA strains

EGCG exposure particularly altered expression patterns of the analyzed genes with higher sensible phenotypes when comparing with the most resistant strains.

Regarding MSSA selected strains (Figure 1 A), MB10 strain was considered the most resistant due to the fact that, like the other strains was sensible to imipenem, tetracycline and gentamicin, but resistant to amoxicillin and, after EGCG exposure, no reversion was observed in the phenotype. Conversely MB17 strain was sensible to all assessed antibiotics and after EGCG exposure we observed a significant increase in transcriptional expression of all analyzed genes, namely $s p d C$, WalR and $\operatorname{orfx}(4.98 \pm 1.14,17.29 \pm 1.9,19.21 \pm 1.99, \mathrm{p}<0.01$ respectively). Also, MB6 with total reversion of amoxicillin resistance $s p d C$, WalR and orf $x$ mRNA were upregulated $(1.62 \pm 0.26,1.85 \pm 0.1,1.5 \pm 0.1$, $\mathrm{p}<0.01$ respectively). On the other hand, after EGCG exposure CC4 strains which presented a reversion of the resistant phenotype for amoxicillin up to $50 \mu \mathrm{g} / \mathrm{ml}$, a downregulation of spdC expression was observed $(-10.865 \pm 0.16$ $\mathrm{p}<0.001)$ and an increase in WalR and orf $x$ mRA levels $(7.88 \pm 0.1 p<0.001$ and $3.9 \pm 0.2 p<0.01$, respectively).

In MRSA selected strains the same pattern was observed (Figure 1 B). For MRSA strains VFXB14 was selected for comparation as it presented the same resistant phenotype as MB10 namely, sensible to imipenem, tetracycline and gentamicin, but maintained resistance to amoxicillin after EGCG exposure. In concordance with MSSA strains, MB5 strain, the most sensible selected MRSA which was sensible to all antibiotics. Transcriptional analysis demonstrated an increase in mRNA levels of $s p d C$ and orfx $(4.74 \pm 0.14,4.23 \pm 0.65, p<0.01$ respectively $)$ and more significantly $\operatorname{WalR}(6.8 \pm 0.28, \mathrm{p}<0.01)$.

On the other hand, in MB2 strains which presented total reversion of amoxicillin resistance only $s p d C$, was significantly upregulated $(20.7 \pm 6.4 \mathrm{p}<0.001)$.

In MB12 strain analysis, which was initially resistant to imipenem but the phenotype was reversed after EGCG exposure and no effect was reported for the amoxicillin resistance $s p d C$, WalR and orf $x$ genes were upregulated (2.74 $\pm 0.24,2.4 \pm 0.12,3.86 \pm 0.22, p<0.01$ respectively). Additionally, VFXB16 strain which presented a reversion of the resistant phenotype for amoxicillin up to $100 \mu \mathrm{g} / \mathrm{ml}$ of EGCG exposure also presented increased mRNA levels of $s p d C$, WalR and orf $x(3.43 \pm 0.13, p<0.01,3.15 \pm 0.17,5.99 \pm 0.14, p<0.001$ respectively $)$. 
Transcriptional analysis performed in the selected strains after 24h of subculture with no EGCG exposure, demonstrated the overall maintenance of the expression patterns observed immediately after EGCG exposure (Figure 2). CC4 strain maintained the downregulation of $s p d C$ expression $(-10.69 \pm 0.29 p<0.01)$ and the WalR and orf $x$ upregulation $(0.73 \pm 0.17 p<0.01$ and $3.9 \pm 0.15 p<0.001$, respectively). However, in MB6 only orf $x$ mRNA upregulation was maintained $(1.77 \pm 0.17, \mathrm{p}<0.001)$. Additionally, in MB17 strain the increase in transcriptional expression WalR and orf $x$ was not altered $(4.48 \pm 2.55,3.23 \pm 0.35, p<0.01$ respectively) but a downregulation of $s p d C$ expression was reported $(-7.35 \pm 0.16, p<0.01)$ (Figure $2 \mathrm{~A})$.

Regarding MRSA strains transcriptional analysis, for MB2 strain we observed the stability of spdC, upregulation $(13.1 \pm 12 p<0.001)$ and additionally, VFXB16 strain also maintained the previous pattern with associated upregulation of $s p d C$, WalR and orf $x$ mRNA levels $(2.11 \pm 0.24, p<0.01,3.02 \pm 0.12, p<0.015 .99 \pm 0.01, p<0.001$ respectively). Moreover, MB5, the most sensible selected MRSA strain, also maintained the upregulation of WalR and orf $x(1.06 \pm$ $0.07,4.47 \pm 0.22, \mathrm{p}<0.001$ respectively) (Figure $2 \mathrm{~B}$ ).

However, conversely in MB12 strain transcriptional analysis, spdC, WalR and orf $x$ genes were downregulated (-3.47 \pm $0.1, p<0.01-3.35 \pm 0.007, p<0.001-2.45 \pm 0.15, p<0.01$ respectively).

\subsection{EGCG oral intake eliminated MRSA phenotype in nasal colonization in the community}

Analyzed data from the performed interventional, uncontrolled, prospective, longitudinal and of individual analysis study demonstrated that all samples presented normal commensal flora of gram positive coccus Staphylococcus spp. and Streptococcus spp. We identified a prevalence of S. aureus 33.3\% in which $70 \%$ were MSSA (23.3\% total) and $30 \%$ MRSA ( $10 \%$ total) at the beginning of the study (Table 6). After 90 days of $225 \mathrm{mg}$ EGCG oral exposure, S. aureus prevalence is maintained (36.6\%) however, regarding MSSA strains, $50 \%$ of the colonized individuals maintained the colonization, $10 \%$ eliminated the colonization and 3 new colonization's were observed, while in MRSA $33.3 \%$ of the colonized individuals eliminated the bacteria while in $66.6 \%$ the resistance phenotype was reversed (Table 6).

Table 6 - MSSA and MRSA prevalence (\%) in commensal flora of the participants enrolled in the study before (T0) and after (T90) 90 days of 225mg EGCG oral exposure. 


\begin{tabular}{|c|c|c|}
\hline ID & T0 prevalence & T90 prevalence \\
\hline 1 & MSSA, Staphylococcus spp. and Streptococcus spp & MSSA, Staphylococcus spp. and Streptococcus spp \\
\hline 2 & Staphylococcus spp. and Streptococcus spp & Staphylococcus spp. and Streptococcus spp \\
\hline 3 & Staphylococcus spp. and Streptococcus spp & Staphylococcus spp. and Streptococcus spp \\
\hline 4 & Staphylococcus spp. and Streptococcus spp & Staphylococcus spp. and Streptococcus spp \\
\hline 5 & MSSA, Staphylococcus spp. and Streptococcus spp & Staphylococcus spp. and Streptococcus spp \\
\hline 6 & Staphylococcus spp. and Streptococcus spp & Staphylococcus spp. and Streptococcus spp \\
\hline 7 & Staphylococcus spp. and Streptococcus spp & MSSA, Staphylococcus spp. and Streptococcus spp \\
\hline 8 & Staphylococcus spp. and Streptococcus spp & MSSA, Staphylococcus spp. and Streptococcus spp \\
\hline 9 & Staphylococcus spp. and Streptococcus spp & Staphylococcus spp. and Streptococcus spp \\
\hline 10 & Staphylococcus spp. and Streptococcus spp & MSSA, Staphylococcus spp. and Streptococcus spp \\
\hline 11 & Staphylococcus spp. and Streptococcus spp & Staphylococcus spp. and Streptococcus spp \\
\hline 12 & Staphylococcus spp. and Streptococcus spp & Staphylococcus spp. and Streptococcus spp \\
\hline 13 & Staphylococcus spp. and Streptococcus spp & Staphylococcus spp. and Streptococcus spp \\
\hline 14 & Staphylococcus spp. and Streptococcus spp & Staphylococcus spp. and Streptococcus spp \\
\hline 15 & Staphylococcus spp. and Streptococcus spp & Staphylococcus spp. and Streptococcus spp \\
\hline 16 & MSSA, Staphylococcus spp. and Streptococcus spp & MSSA, Staphylococcus spp. and Streptococcus spp \\
\hline 17 & MRSA, Staphylococcus spp. and Streptococcus spp & MSSA, Staphylococcus spp. and Streptococcus spp \\
\hline 18 & MRSA, Staphylococcus spp. and Streptococcus spp & Staphylococcus spp. and Streptococcus spp \\
\hline 19 & MRSA, Staphylococcus spp. and Streptococcus spp & MSSA, Staphylococcus spp. and Streptococcus spp \\
\hline 20 & Staphylococcus spp. and Streptococcus spp & Staphylococcus spp. and Streptococcus spp \\
\hline 21 & MSSA, Staphylococcus spp. and Streptococcus spp & MSSA, Staphylococcus spp. and Streptococcus spp \\
\hline 22 & Staphylococcus spp. and Streptococcus spp & Staphylococcus spp. and Streptococcus spp \\
\hline 23 & Staphylococcus spp. and Streptococcus spp & Staphylococcus spp. and Streptococcus spp \\
\hline 24 & Staphylococcus spp. and Streptococcus spp & Staphylococcus spp. and Streptococcus spp \\
\hline 25 & MSSA, Staphylococcus spp. and Streptococcus spp & MSSA, Staphylococcus spp. and Streptococcus spp \\
\hline 26 & MSSA, Staphylococcus spp. and Streptococcus spp & MSSA, Staphylococcus spp. and Streptococcus spp \\
\hline 27 & Staphylococcus spp. and Streptococcus spp & Staphylococcus spp. and Streptococcus spp \\
\hline 28 & MSSA, Staphylococcus spp. and Streptococcus spp & MSSA, Staphylococcus spp. and Streptococcus spp \\
\hline 29 & Staphylococcus spp. and Streptococcus spp & Staphylococcus spp. and Streptococcus spp \\
\hline 30 & Staphylococcus spp. and Streptococcus spp & Staphylococcus spp. and Streptococcus spp \\
\hline
\end{tabular}

\section{Discussion}

Page $11 / 19$ 
Over the past decade, there has been a concerning increase in resistance to antibiotics from microorganisms that are pathogenic to humans. This has become a global problematic with hazardous health consequences, but also with consequences for the economy, which has raised some concerns regarding the prescription and intensive use of antibiotics $(2,3,29,30)$. Currently, infections caused by $S$. aureus are no longer an exclusive problem associated with hospital environments and are becoming an emerging problem in the community (31). Thus, several studies in the scientific community have focused in the development of new and alternative therapeutic approaches based on the use of natural products or compounds with therapeutic properties and potential $(16,32,33)$. The use of catechin EGCG in green tea is one of these approaches, as well as the study of its beneficial properties (34-36). It has been proven in several studies that EGCG has anti-infectious properties against Gram-negative and Gram-positive bacteria, in some fungi and viruses $(16,37,38)$.

Here, we assessed the prevalence of $S$. aureus strains sensible and resistant to methicillin in health and the potential of EGCG as a new compound with antimicrobial properties and synergistic potential with common antibiotics as well as its impact on epigenetic and resistance modulator genes in strains with divergent phenotypes of resistance.

Data analysis revealed that health care occupational exposure lead to a prevalence of $42.1 \%$ of $S$. aureus colonization (18.4\% MSSA and 23.7\% MRSA), which is concerningly high. Previous studies carried out in Portugal emphasize that the main mode of transmission of MRSA is through the hands, with the absence of proper hand hygiene being the most common mode of transmission (39) and since health professionals are exposed for patients who may be contaminated, non-compliance with hygiene rules can be a way of spreading MRSA inside and outside the hospital environment, but also in the community (40). As previously mentioned, $S$. aureus has the ability to colonize different areas of the human body, with a preference for the nasopharynx (41) and the ability to spread as well as being transmitted by direct contact (mainly by hands) or indirect (contaminated surfaces) (42). One of its fundamental biological characteristics is the ability to colonize the healthy population asymptomatically (asymptomatic carrier), thus assuming an important role in spreading to other areas of the body, to other people and even contaminating food and surfaces during handling (42). This colonization is considered a risk factor for the onset of infections by $S$. aureus, often combined with methicillin resistance - MRSA, increasing the risk of clinical disease $(42,43)$. Thus, our results sustain the prerogative that exposure to bioaerosols particularly at workplaces can represent a health hazard and potentially result in infectious disease (44) which is concerning either for workers and for the spread of these microorganisms in the community. Currently, there are several antibiotics with different modes of action, namely inhibition of cell wall synthesis, inhibition of protein synthesis, inhibition of nucleic acid synthesis, destruction of cell membrane function, and inhibition of metabolism available for therapeutic approaches. In this study, the strains were subjected to the action of four antibiotics: imipenem $10 \mu \mathrm{g}$, tetracycline $30 \mu \mathrm{g}$, gentamicin $30 \mu \mathrm{g}$ and amoxicillin $25 \mu \mathrm{g}$, with EGCG in different concentrations, in order to assess synergism and be able to define its resistance phenotype. Blactams such as amoxicillin, and imipenem (belonging to the carbapenem subgroup) are able to interfere with the bacterial cell wall synthesis leading to cell lysis $(5,14,17,45,46)$.

In this study we clearly demonstrated the synergism between EGCG and strains with resistance phenotype as we verified that there were changes in the phenotype from resistant to sensible in some of the studied strains associated with imipenem and amoxicillin. Although only one isolated strain showed phenotype of resistance to imipenem, it was reverted when exposed to different concentrations of EGCG. Regarding amoxicillin, of the strains tested, 10 had an initial resistance phenotype, however when exposed to different concentrations of EGCG, 3 strains reversed the resistant to sensible phenotype, one reversed the phenotype in concentrations of $250 \mu \mathrm{g} / \mathrm{ml}, 100 \mu \mathrm{g} / \mathrm{ml}$ and $50 \mu \mathrm{g} /$ $\mathrm{ml}$, but at the concentration of $25 \mu \mathrm{g} / \mathrm{ml}$ the resistance phenotype remained. Additionally, in two strains only at the concentrations of $250 \mu \mathrm{g} / \mathrm{ml}$ and $100 \mu \mathrm{g} / \mathrm{ml}$ of EGCG did a phenotype reversal occur. Although the number of strains collected from the community is reduced, we were able to verify that for antibiotics that have a cell wall synthesis

Page $12 / 19$ 
mechanism of action, there was a synergism with EGCG, which is in line with previous studies $(13,16,38,47)$. Moreover, time exposure also seems to be critical for EGCG interaction as we observed that there are differences in MSSA and MRSA strains, between $18 \mathrm{~h}, 24 \mathrm{~h}$ and $48 \mathrm{~h}$ of exposure for imipenem and amoxicillin.

Our results demonstrate that divergent resistant phenotypes are associated with different transcriptional expression of epigenetic modulators, which was particularly noticeable in the most sensible strains. Regarding WalR, it reaches its highest transcription levels in the most sensible strains, obtaining 17.29 \pm 1.9 fold-change in MSSA and $6.8 \pm 0.28$ fold-change for MRSA. The WalKR two-component system is essential for $S$. aureus viability, actively participating in the cell wall metabolism $(23,48)$. WalR positively regulates autolysis, biofilm production and alpha-hemolytic activity and positively regulates relevant virulence genes, including the spdC gene $(22,23)$. Activation of WalR decreases $S$. aureus virulence, inducing an early triggering of the host's inflammatory response, including neutrophil recruitment and increased cytokine levels thus, leading to a rapid bacterial clearance and lowered virulence (23). In the present study, EGCG had a great influence in WalR transcription levels in the most sensible strains, which may contribute for their lower virulence. On the other hand, spdC gene was the only one downregulated, namely in CC4, a MSSA strain, with amoxicillin resistance phenotype reverted with EGCG concentration up to $50 \mu \mathrm{g} / \mathrm{ml}$. Reduced $s p d C$ transcription levels are associated with a lowered virulence and increased sensitivity to $\beta$-lactam antibiotics (22), however, we could not find a significant association of the $s p d C$ gene transcription levels and the resistance phenotype. Moreover, in MSSA strains we observed an increased expression of the staphylococcal ribosomal methyltransferase, particularly in sensible strains, reaching a $19.21 \pm 1.99$-fold change. Strains with resistant phenotype reversion in vitro also revealed a correlation between EGCG exposure and orf $x$ transcription levels, from $1.5 \pm 0.1$ to $6 \pm 0.14$ fold change. This expression decreases after subculture in the absence of EGCG, which suggests a direct exposure effect, however maintaining the overall patterns previously observed. Orf $x$ is conserved among all staphylococci, and it is constitutively produced during growth $(21,49)$. Recently, this methyltransferase has been of particular interest due to its the insertion site in the $\mathrm{SCCmec}$ mobile genomic island within its $\mathrm{C}$ terminus, in the attachment site (attB) $(21,50,51)$. Boundy et. coworkers monitored orf $x$ by Western blotting, showing that the insertion of SCCmecinto at site had no effect on gene expression or on protein production $(21,51)$. Thus, $S$. aureus susceptibility to oxacillin, mediated by the mecA gene in SCCmec is not affected by inactivation of orf $x$, which agrees to the results obtained for both MSSA and MRSA $(50,51)$. On the other hand, orf $x$ gene product has been assumed to play a key role in bacterial growth and survival, as it is present in all sequenced coagulase-positive or coagulase-negative staphylococcal genomes $(21,49,52)$. Orf $x$ methylate $70 \mathrm{~S}$ ribosomes, constituting a staphylococcal ribosomal methyltransferase of RImH type (49). Relevantly, ribosome methylation in bacteria provides either moderate resistance to antibiotics or, on the contrary, determine susceptibility to antibiotics, thus affecting bacterial adaptation and resistance $(21,49,52)$. Our data suggests that orfx-mediated ribosomal methylation is affected by EGCG exposure, playing an important role in determining phenotype resistance reversion as an epigenetic modulator. In recent studies, Kitichalermkiat et. coworkers conducted a microarray analysis on $S$. aureus treated with or without 500 mg/L of EGCG in which differentially expressed genes were identified and their changes at the transcription level were confirmed using real-time qPCR (53). Similarly, to the present study, EGCG treatment resulted in increased transcription expression (75 genes) of genes particularly related to membrane transport and decreased transcription (72 genes) in genes involved in toxin production and stress response (53). Additionally, cellular membrane potential assessment also concluded that EGCG markedly decreased membrane potential, which is suggestive of cell membrane damage (53).

Overall our results clearly demonstrate that EGCG exposure is able to alter expression patterns of key epigenetic and drug response genes in $S$. aureus with associated divergent resistant profiles and should be further investigated potentially as a natural antimicrobial agent and or a therapeutic adjuvant.

Page 13/19 


\section{Conclusions}

Overall, this study allowed to sustain the antimicrobial and synergistic potential of EGCG with antibiotics that inhibit cell wall synthesis (imipenem and amoxicillin) in strains of $S$. aureus, particularly MSSA, and the concerning high colonization prevalence of these pathogens.

This work also demonstrated that divergent resistant phenotypes of $S$. aureus strains are associated with differential expression of epigenetic and drug resistance modulator genes which indicates a clear modulator effect induced by EGCG exposure, and corroborates the potential of EGCG for antimicrobial and / or therapeutic adjuvant treatment against antibiotic-resistant microorganisms.

\section{Abbreviations}

Epigalocatenin-3-gallate (EGCG), Health Care-associated Methicillin-Resistant Staphylococcus aureus infections (HAMRSA, Methicillin-Resistant Staphylococcus aureus (MRSA) are Methicillin-sensible Staphylococcus aureus (MSSA), European Committee on Antimicrobial Susceptibility Testing (EUCAST), Real-Time Quantitative Reverse Transcription (RT-qPCR), World Health Organization (WHO), Staphylococcal Cassette Chromosome mec complex (SCCmec).

\section{Declarations}

Author Contributions: Conceptualization, ER., methodology, A.M, M.D., T.P., A.Z.., validation, E.R. and M.B., formal analysis, E.R., resources, E.R. and M.B., writing-original draft preparation, A.M., T.P. and E.R., writing-review and editing, E.R., supervision, E.R., project administration, E.R., funding acquisition, E.R. All authors have read and agreed to the published version of the manuscript.

Funding: This work was supported by Instituto Politécnico de Lisboa, Lisbon, Portugal for funding the Project " Resistance modulation and epigenetic divergence in resistant phenotypic profiles of Staphylococcus Aureus" (IPL/2020/ EpiResistanceSA_ESTeSL). H\&TRC authors gratefully acknowledge the FCT/MCTES national support through the UIDB/05608/2020 and UIDP/05608/2020.

Institutional Review Board Statement: The study was conducted according to the guidelines of the Declaration of Helsinki, and approved by the Institutional Ethics Committee of Escola Superior de Tecnologia da Saúde ref: CEESTeSL-Nº.18-2019 and CE-ESTeSL-Nº. 20-2020.

Informed Consent Statement: Informed consent was obtained from all subjects involved in the study.

Conflicts of Interest: The authors declare no conflict of interest

\section{References}

1. World Health Organization (WHO). Antimicrobial resistance: fact sheet no 194. Genebra: WHO. 2016.

2. Morris AK, Masterton RG. Antibiotic resistance surveillance: action for international studies. J Antimicrob Chemother. 2002,49(1):7-10. 
3. Smith DL, Harris AD, Johnson J a, Silbergeld EK, Morris JG. Animal antibiotic use has an early but important impact on the emergence of antibiotic resistance in human commensal bacteria. Proc Natl Acad Sci U S A. 2002,99:6434-9.

4. Lakhundi S, Zhang K. Methicillin-Resistant Staphylococcus aureus: Molecular Characterization, Evolution, and Epidemiology. Clin Microbiol Rev. 2018,31(4):1-103.

5. Peacock SJ, Paterson GK. Mechanisms of Methicillin Resistance in Staphylococcus aureus. Annu Rev Biochem. 2015,84:577-601.

6. Turner N, Sharma-Kuinkel B, Maskarinec S, Eichenberger E. Methicillin-resistant Staphylococcus aureus: an overview of basic and clinical research. Nat Rev Microbiol. 2019,17(February):203-18.

7. Rolo J, Miragaia M, Turlej-Rogacka A, Empel J, Bouchami O, Faria NA, et al. High genetic diversity among community-associated staphylococcus aureus in europe: Results from a multicenter study. PLoS One. 2012,7(4).

8. Klevens RM, Morrison MA, Nadle J, Petit S, Gershman K, Ray S, Harrison LH, Lynfield R, Dumyati G, Townes JM, Craig AS, Zell ER, Fosheim GE, McDougal LK, Carey RB FSABC surveillance (ABCs) MI. Invasive methicillinresistant Staphylococcus aureus infections in the United States. JAMA. 2007,298(15):1763-71.

9. Jordan D, Simon J, Fury S, Moss S, Giffard P, Maiwald M, et al. Carriage of methicillin-resistant Staphylococcus aureus by veterinarians in Australia. Aust Vet J. 2011,89(5):152-9.

10. Laux C, Peschel A, Krismer B. Staphylococcus aureus Colonization of the Human Nose and Interaction with Other Microbiome Members. Microbiol Spectr. 2018,7(2):1-10.

11. Al-tamimi M, Himsawi N, Abu-raideh J, Jazar DA, Al-jawaldeh H, Mahmoud SAH, et al. Nasal colonization by methicillin-sensible and methicillin-resistant Staphylococcus aureus among medical students. J Infect Dev Ctries. 2018,2(5):326-35.

12. Mehraj J, Witte W, Akmatov M, Layer F, Werner G, Krause G. Epidemiology of Staphylococcus aureus Nasal Carriage Patterns in the Community. Curr Top Microbiol Immunol. 2016,398:55-87.

13. Zhao W, Hu Z, Okubo S, Hara Y. Mechanism of Synergy between Epigallocatechin Gallate and $\beta$-Lactams against Methicillin-Resistant Staphylococcus aureus. Antimicrob Agents Chemother. 2001,45(6):1737-42.

14. Hu Z, Zhao W, Asano N, Yoda Y, Hara Y, Shimamura T. Epigallocatechin Gallate Synergistically Enhances the Activity of Carbapenems against Methicillin-Resistant Staphylococcus aureus. Antimicrob Agents Chemother. 2002,46(2):558-60.

15. Roccaro AS, Blanco AR, Giuliano F, Rusciano D, Enea V. Epigallocatechin-Gallate Enhances the Activity of Tetracycline in Staphylococci by Inhibiting Its Efflux from Bacterial Cells. Antimicrob Agents Chemother. 2004,48(6):1968-73.

16. Steinmann J, Buer J, Pietschmann T, Steinmann E. Anti-infective properties of (EGCG), a component of green tea. Br J Pharmacol. 2013,168:1059-73.

17. Hu Z, Zhao W, Hara Y, Shimamura T. Epigallocatechin gallate synergy with ampicilin/sulfabactam against 28 clinical isolates of methicillin-resistant Staphylococcus aureus. J Antimicrob Chemother. 2001,48:361-4.

18. Araújo R, Ramalhete L, Paz H Da, Ribeiro E, Calado CRC. A Simple, Label-Free, and High-Throughput Method to Evaluate the Epigallocatechin-3-Gallate Impact in Plasma Molecular Profile. High-Throughput. 2020,9(9):1-12.

19. Vahid F, Zand H, Nosrat-Mirshekarlou E, Najafi R, Hekmatdoost A. The role dietary of bioactive compounds on the regulation of histone acetylases and deacetylases: A review. Gene. 2015,562(1):8-15.

20. Negri A, Naponelli V, Rizzi F, Bettuzzi S. Molecular Targets of Epigallocatechin-Gallate (EGCG): A Special Focus on Signal Transduction and Cancer. Nutrients. 2018,10(1936).

Page 15/19 
21. Boundy S, Safo MK, Wang L, Musayev FN, Farrell HCO, Rife JP, et al. Characterization of the Staphylococcus aureus rRNA Methyltransferase Encoded by orfx, the Gene Containing the Staphylococcal Chromosome Cassette mec ( SCC mec ) Insertion Site *. J Biol Chem. 2013,288(1):132-40.

22. Poupel O, Proux C, Jagla B, Msadek T, Dubrac S. SpdC, a novel virulence factor , controls histidine kinase activity in Staphylococcus aureus. PLoS Pathog. 2018,14(3):1-32.

23. Delauné A, Dubrac S, Blanchet C, Poupel O, Mäder U, Hiron A, et al. The WalKR System Controls Major Staphylococcal Virulence Genes and Is Involved in Triggering the Host Inflammatory Response. Infect Immun. 2012,80(10):3438-53.

24. Ji Q, Chen PJ, Qin G, Deng X, Hao Z, Wawrzak Z, et al. Structure and mechanism of the essential two-component signal-transduction system WalKR in Staphylococcus aureus. Nat Commun. 2016,

25. Wu S, Lin K, Liu Y, Zhang H, Lei L. Two-component signaling pathways modulate drug resistance of Staphylococcus aureus (Review). Biomed REPORTS. 2020,13(5):1-5.

26. NCT00942422. Green Tea Extract in Treating Patients With Monoclonal Gammopathy of Undetermined Significance and/or Smoldering Multiple Myeloma.

27. Chow HS, Cai Y, Hakim IA, Crowell JA, Shahi F, Brooks CA, et al. Pharmacokinetics and Safety of Green Tea Polyphenols after Multiple-Dose Administration of Epigallocatechin Gallate and Polyphenon E in Healthy Individuals. Clin Cancer Res. 2003,9:3312-9.

28. EUCAST. EUCAST Clinical breakpoint tables for interpretation of MICs and zone diameters. 2020.

29. World Health Organization (WHO). Antimicrobial resistance. 2020.

30. Khasru A, Saha K, Rahman J, Ara R, Rahman A, Kanti S, et al. Antibacterial activities of green tea crude extracts and synergistic effects of epigallocatechingallate (EGCG) with gentamicin against MDR pathogens. Heliyon. 2019,5(July).

31. Gelatti LC, Becker AP, Bonamigo RR, D’Azevedo PA. Methicillin-resistant Staphylococcus aureus: emerging community dissemination. An Bras Dermatol. 2009,84(5):501-6.

32. Betts JW, Sharili AS, Phee LM, Wareham DW. In Vitro Activity of Epigallocatechin Gallate and Quercetin Alone and in Combination versus Clinical Isolates of Methicillin-Resistant Staphylococcus aureus. J Nat Prod. 2015,3-6.

33. Haghjoo B, Lee LH, Habiba U, Tahir H, Olabi M, Chu T. The synergistic effects of green tea polyphenols and antibiotics against potential pathogens. Adv Biosci Biotechnol. 2013,4:959-67.

34. Chu C, Deng J, Man Y, Qu Y. Green Tea Extracts Epigallocatechin-3-gallate for Different Treatments. Biomed Res Int. 2017,

35. Singh BN, Shankar S, Srivastava RK. Green tea catechin, epigallocatechin-3-gallate (EGCG): mechanisms, perspectives and clinical applications. Biochem Pharmacol. 2011,82(12):1807-21.

36. Martini N. Green Tea. J PRIM Heal CARE. 2016,8(4):381-2.

37. Bakkir LK, Yassen RT, Mustaffa RM. In vitro and in vivo study of green and black tea antimicrobial activity on methicillin resistant Staphylococcus aureus. Bas J Vet Res. 2011,10(2).

38. Reygaert WC. Green Tea Catechins: Their Use in Treating and Preventing Infectious Diseases. Biomed Res Int. 2018,(9105261).

39. Mondal H, Gupta I, Nandi P, Ghosh P, Chattopadhyay S, Mitra GD. Nasal Screening of Healthcare Workers for Nasal Carriage of Methicillin Resistance Staphylococcus Aureus, Vancomycin Resistance Staphylococcus Aureus and Prevalence of Nasal Colonization with Staphylococcus Aureus in Burdwan Medical College and Hospital. Int J Contemp Med Res. 2016,3(11):3342-6. 
40. Dulon M, Peters C, Schablon A, Nienhaus A. MRSA carriage among healthcare workers in non-outbreak settings in Europe and the United States: a systematic review. BMC Infect Dis. 2014,14(363).

41. Mainous III AG, Hueston WJ, Everett CJ, Diaz VA. Nasal Carriage of Staphylococcus aureus and MethicillinResistant S aureus in the United States, 2001-2002. Ann Fam Med. 2006,4(2):2001-2.

42. Ribeiro E, Clérigo A. Assessment of staphylococcus aureus colonization in bakery workers.pdf. Vertentes e Desafios Segurança. 2017,

43. Ghasemzadeh-Moghaddam H, Neela V, Wamel W Van, Hamat RA, Shamsudin M, Hussin NSC, et al. Nasal carriers are more likely to acquire exogenous Staphylococcus aureus strains than non-carriers. Clin Microbiol Infect. 2015,21(11):998.e1-998.e7.

44. Walser SM, Gerstner DG, Brenner B, Bünger J, Eikmann T, Janssen B, et al. Evaluation of exposure-response relationships for health effects of microbial bioaerosols - A systematic review. Int J Hyg Environ Health. 2015,218(7):577-89.

45. Foster TJ. Antibiotic resistance in Staphylococcus aureus. Current status and future prospects. FEMS Microbiol Rev. 2017,(December 2016):1-20.

46. Fernandes R, Amador P, Prudêncio C. b-Lactams: chemical structure , mode of action and mechanisms of resistance. 2013,

47. Yam TS, Hamilton-Miller JMT, Shah S. The effect of a component of tea (Camelia sinensis) on methicilin resistance, PBP2' synthesis and b-lactamase production in Staphylococcus aureus. J Antimicrob Chemother. 1998,42:211-6.

48. Ji Q, Chen PJ, Qin G, Deng X, Hao Z, Wawrzak Z, et al. Structure and mechanism of the essential two-component signal transduction system WalKR in Staphylococcus aureus. Nat Commun. 2016,7(Mar).

49. Bitrus AA, Zunita Z, Khairani-Bejo S, Othman S, Ahmad Nadzir NA. Staphylococcal cassette chromosome mec (SCCmec) and characterization of the attachment site (attB) of methicillin resistant Staphylococcus aureus (MRSA) and methicillin susceptible Staphylococcus aureus (MSSA) isolates. Microb Pathog [Internet]. 2018,123(July):323-9. Available from: https://doi.org/10.1016/j.micpath.2018.07.033

50. Miragaia M. Factors contributing to the evolution of Meca-mediated $\beta$-lactam resistance in staphylococci: Update and new insights from whole genome sequencing (WGS). Front Microbiol. 2018,9(NOV):1-16.

51. Noto MJ, Kreiswirth BN, Monk AB, Archer GL. Gene acquisition at the insertion site for SCCmec, the genomic island conferring methicillin resistance in Staphylococcus aureus. J Bacteriol. 2008,190(4):1276-83.

52. Osterman IA, Dontsova OA, Sergiev P V. rRNA Methylation and Antibiotic Resistance. Biochem. 2020,85(11):1335-49.

53. Kitichalermkiat A, Katsuki M, Sato J, Sonoda T, Masuda Y, Honjoh K ichi, et al. Effect of epigallocatechin gallate on gene expression of Staphylococcus aureus. J Glob Antimicrob Resist [Internet]. 2020,22:854-9. Available from: https://doi.org/10.1016/j.jgar.2020.06.006

\section{Figures}


MSSA strains

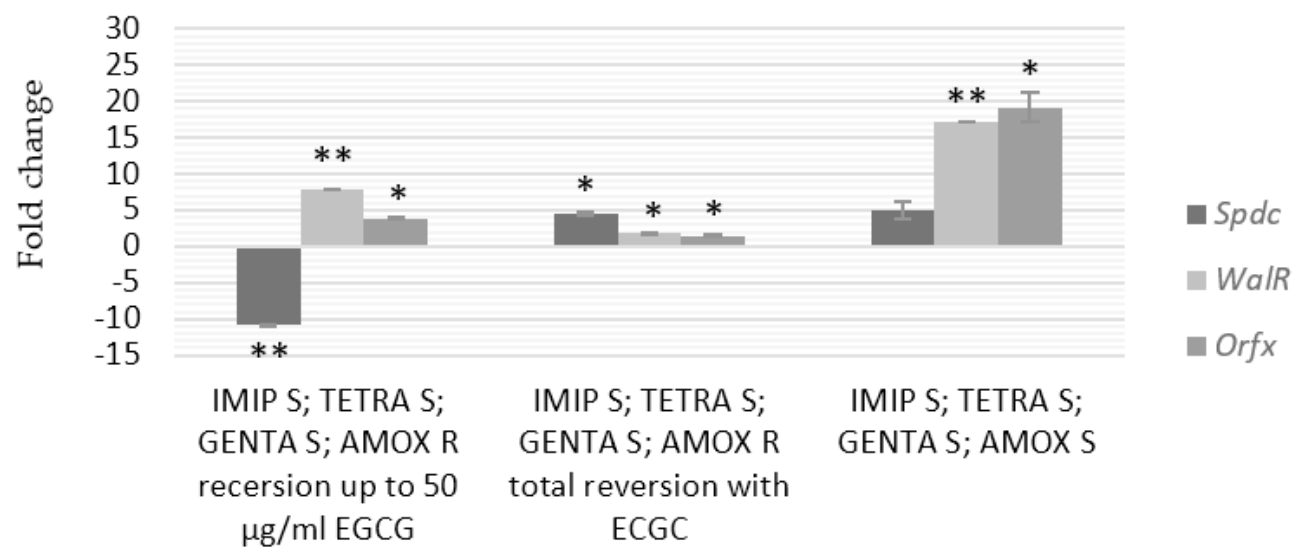

B

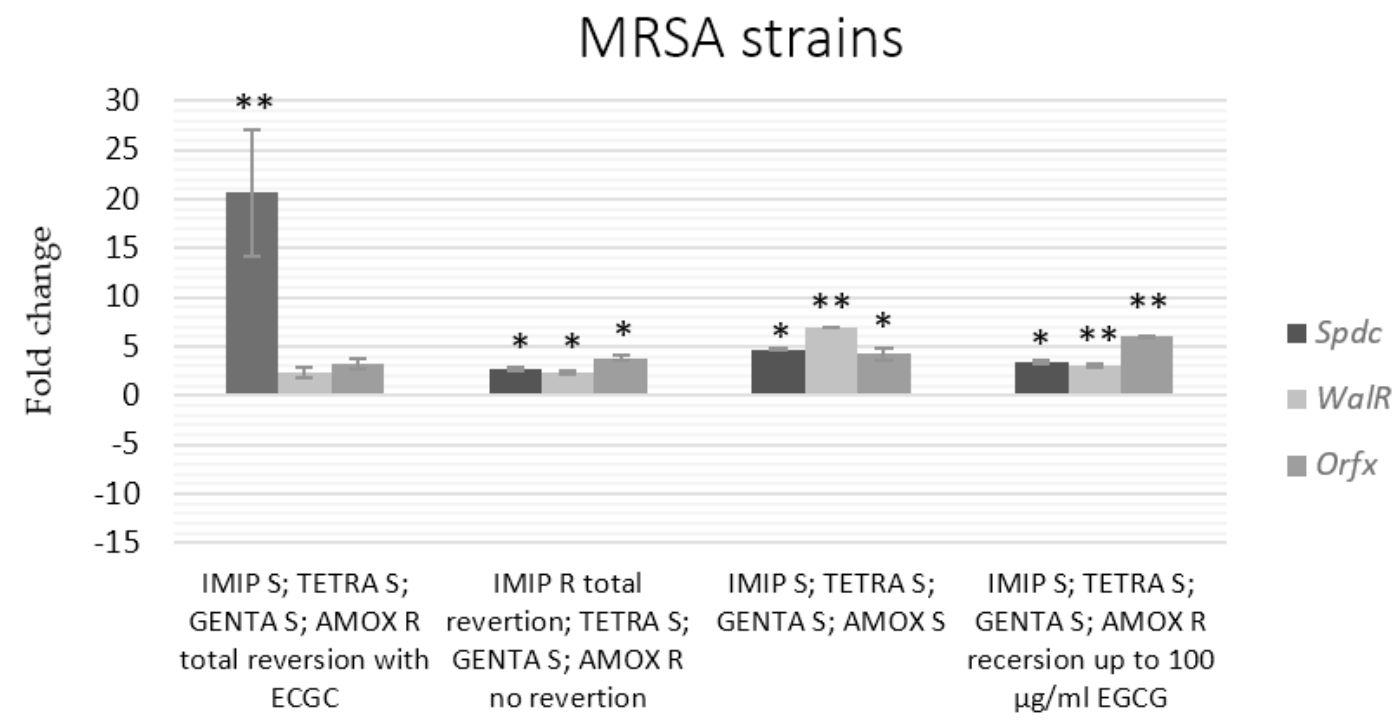

Figure 1

Graphic representation of MSSA (A) and MRSA (B) selected strains with divergent resistance phenotypes after EGCG exposure qRT-PCR results. Data represent the relative expression for the genes: orfx, spdC and WalKR. 16S rRNA was used for normalization. Error bars represent the standard deviation between independent treatments and qRT-PCR replicates. Significant statistical values, which were compared with the most resistant strains and calculated with Student's t-test, are illustrated as: ${ }^{*} \mathrm{p}<0.01$ and ${ }^{* *} \mathrm{p}<0.001$. 


\section{MSSA strains}

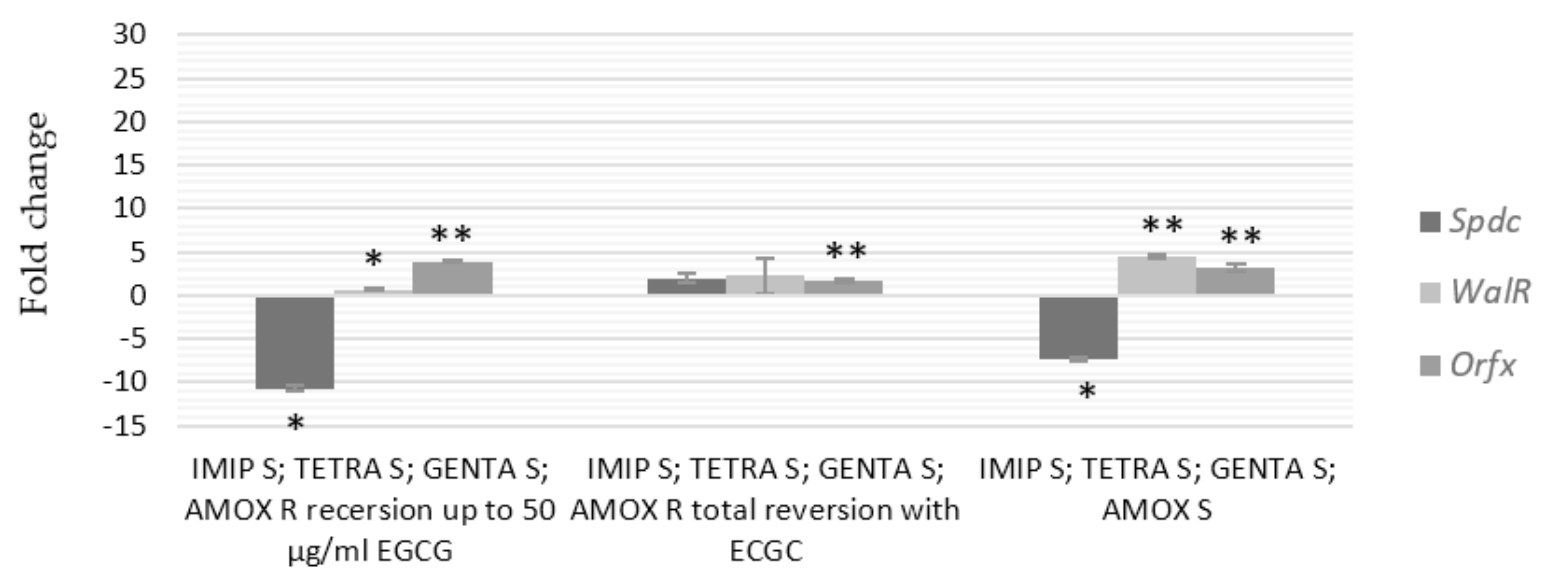

B

\section{MRSA strains}

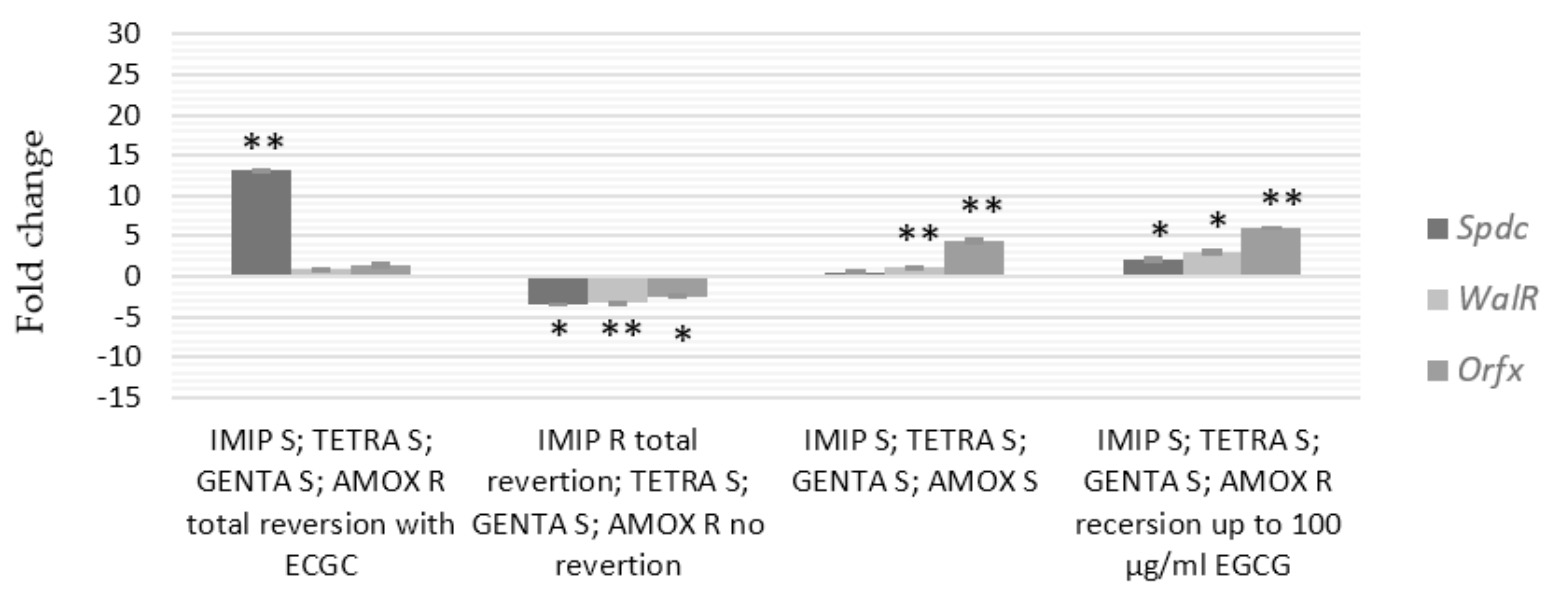

\section{Figure 2}

Graphic representation of MSSA (A) and MRSA (B) selected strains with divergent resistance phenotypes after EGCG exposure, qRT-PCR results after 24h of subculture. Data represent the relative expression for the genes: orfx, spdC and WaIKR. 16S rRNA was used for normalization. Error bars represent the standard deviation between independent treatments and qRT-PCR replicates. Significant statistical values, which were compared with the most resistant strains and calculated with Student's t-test, are illustrated as: ${ }^{*} p<0.01$ and ${ }^{*} p<0.001$. 